\title{
Freezing in finite systems: hard discs in circular cavities
}

\author{
Z T Németh†‡ and H Löwen $\dagger \S$ \\ $\dagger$ Institut für Festkörperforschung, Forschungszentrum Jülich GmbH, D-52425 Jülich, Germany \\ ‡ Veszprémi Egyetem, Fizika Tanszék, Egyetem ut 10, H-8200 Veszprém, Hungary \\ $\S$ Institut für Theoretische Physik II, Heinrich-Heine-Universität Düsseldorf, Universitätsstraße \\ 1, D-40225 Düsseldorf, Germany
}

Received 6 February 1998

\begin{abstract}
Statistical and ergodic properties of a simple model of $N$ hard discs in a circular cavity are investigated by means of analytical considerations and molecular dynamics computer simulations. The model exhibits ergodicity breaking at different densities as well as a kinetic crossover from hydrodynamic relaxation to particle exchange hopping processes. We calculate the associated densities over a large range of $N$. The threshold densities for the kinetic crossover are not correlated with the ergodicity-breaking density. For $N \rightarrow \infty$, the kinetic crossover corresponds to the freezing transition of the bulk system of hard discs. For finite $N$, we found that the crossover densities are smaller than the bulk freezing density. We finally discuss the relevance of our results for the glass and crystallization transitions in porous media.
\end{abstract}

\section{Introduction}

In the usual statistical mechanical definition of phase transitions, the thermodynamic limit is involved, where the system volume $\Omega$ and the number $N$ of particles tend to infinity while the number density $\rho=N / \Omega$ is kept fixed. Emerging non-analyticities of the free energy determine the locations of phase boundaries as a function of the corresponding thermodynamic variables such as temperature $T$, number density $\rho$ and pressure $P$. The result is independent of which ensemble is used. In recent years, it has become popular to study the thermodynamics of finite systems [1]. It is clear that-at least in the general traditional sense-there is no sharp phase transition in a finite system. Moreover, the different ensembles are no longer equivalent. The interesting question, however, is that of whether and how the bulk phase transition manifests itself in a finite system. It is clear that such a 'fingerprint' of a bulk phase transition in a finite system would depend strongly on the system size. A detailed knowledge of the thermodynamics of finite systems is of great interest for various reasons. First, every real system is finite. Second, in a computer simulation there is no obvious way of taking the thermodynamic limit and one has to consider finite-size effects carefully. Third, on confining a system heavily, there emerge quite novel effects not known from the bulk behaviour as far as both structural and dynamical correlations are concerned [2-4]. This was strikingly demonstrated by experiments on the dynamics of molecular liquids confined to porous media—see, e.g., [5, 6]—and on the structure and dynamics of two-dimensional magnetic colloids confined into two-dimensional cavities of different shapes [7].

The aim of this paper is to perform a theoretical study of phase transitions in heavily confined systems consisting of $N$ particles in two-dimensional cavities. In order to address 
principles, we try to keep the model simple. Therefore we have studied a toy model of $N$ two-dimensional hard discs in a hard circular cavity. This model has the advantage that the temperature scales out and the only remaining thermodynamic parameter is the density or the area fraction. The simplicity of the model makes it possible to study ergodicity properties of the finite system as well as to calculate structural and dynamical correlations within the molecular dynamics computer simulations. For increasing density, we find that the system exhibits a crossover from a regime where the long-time dynamics is dominated by hydrodynamic relaxation to another regime where the dynamics is governed by particle exchange hopping processes. The corresponding threshold densities of this kinetic crossover are calculated for different $N$. For large $N$, these densities fall into the coexistence region of the bulk freezing transition of the hard-discs system from a fluid to a hexagonal twodimensional crystal [8]. For finite $N$ the transition densities are significantly smaller than the bulk freezing density. This demonstrates the important influence of the system boundaries on the freezing transition. Our model also exhibits several ergodicity-breaking transitions which can be partially assessed exactly. Interestingly enough, these transition densities do not scale with the densities associated with the dynamical crossover, i.e., in general, they behave differently as functions of the total particle number $N$.

Our paper is organized as follows. In section 2, we introduce our model and discuss simple geometric and ergodic properties. Static quantities are discussed in section 3. It turns out that the static correlations are quite insensitive to the density. Hence we take dynamical correlations as diagnostic tools for a freezing transition and calculate the corresponding transition densities in section 4 . We give our conclusions in section 5.

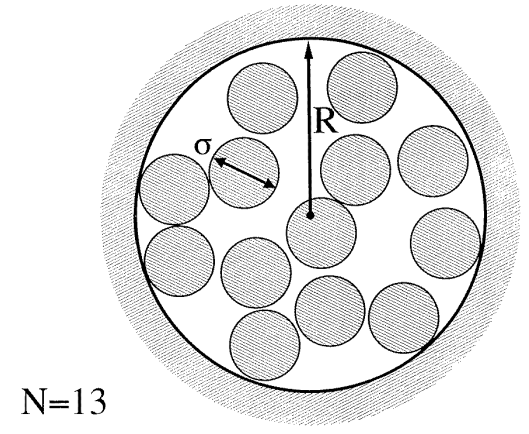

Figure 1. $N$ hard discs in a spherical cavity. $R$ denotes the radius of the cavity; $\sigma$ is the hard-disc diameter.

\section{The model and its properties}

\subsection{The model}

We study a two-dimensional system of $N$ hard discs of diameter $\sigma$ in a circular cavity of radius $R$; see figure 1 . The positions of the disc centres are denoted by $\boldsymbol{r}_{i}(i=1, \ldots, N)$. The origin of our coordinate frame is taken to be the cavity centre. The boundary of the cavity is modelled as a hard wall, i.e. the interaction of a disc with the cavity is given by the external potential

$$
V^{e x t}(r)= \begin{cases}0 & r \leqslant R-\sigma / 2 \\ \infty & r>R-\sigma / 2\end{cases}
$$




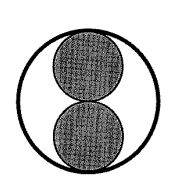

$\mathrm{N}=2$

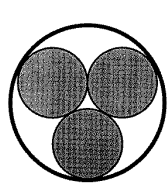

$\mathrm{N}=3$

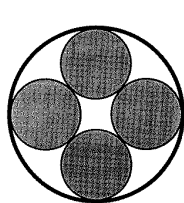

$\mathrm{N}=4$

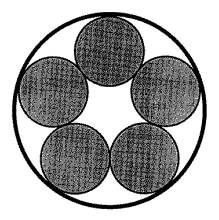

$\mathrm{N}=5$

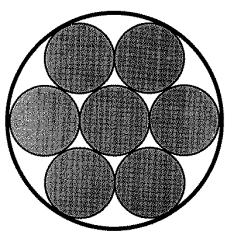

$\mathrm{N}=6,7$

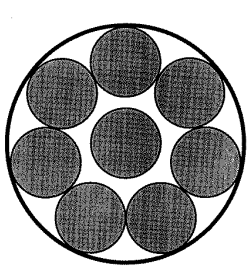

$\mathrm{N}=8$

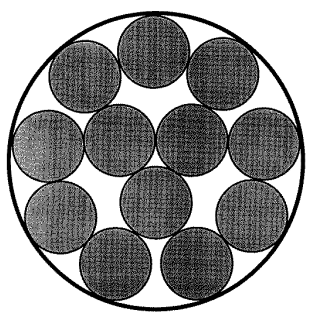

$\mathrm{N}=12$

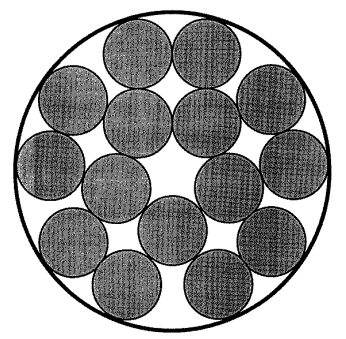

$\mathrm{N}=15$

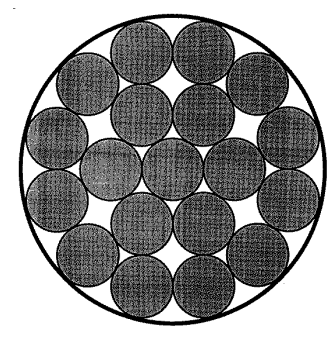

$\mathrm{N}=18,19$

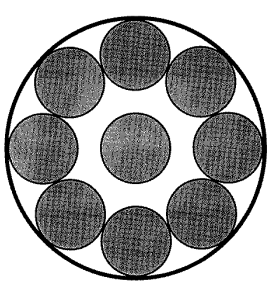

$\mathrm{N}=9$

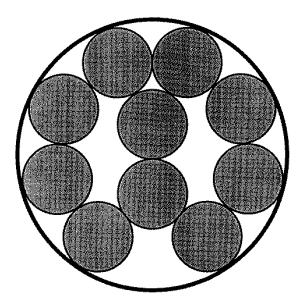

$\mathrm{N}=10$

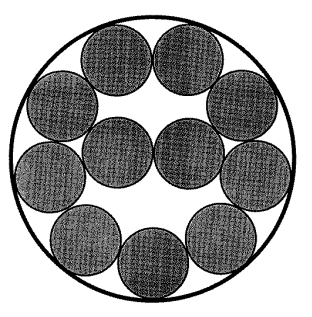

$\mathrm{N}=11$

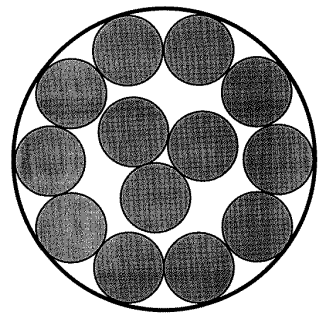

$\mathrm{N}=13$

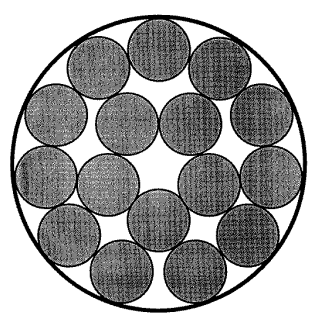

$\mathrm{N}=16$

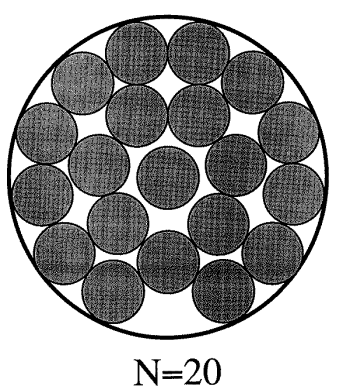

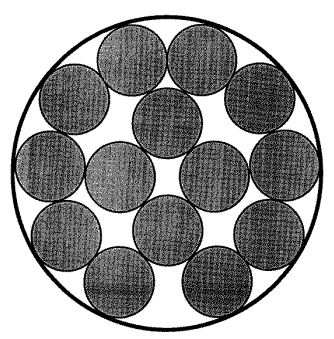

$\mathrm{N}=14$

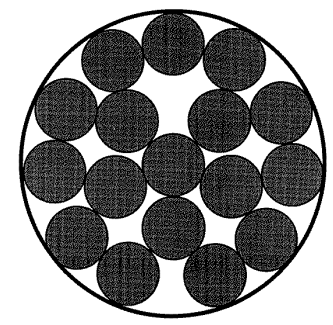

$\mathrm{N}=17$

Figure 2. The close-packed configurations for $N=2-20$. These are rigorously proven only for $N \leqslant 11$. 
where $r$ is the distance from the disc to the origin.

Since only excluded-area interactions are involved, the temperature $T$ scales out as far as static quantities are concerned. It only sets the relevant energy scale $k_{B} T$. The only remaining thermodynamic parameter is the number density per unit area, $\rho=N / A=$ $N / \pi R^{2}$, or the corresponding area fraction, defined by

$$
\eta=N\left(\frac{\sigma}{2 R}\right)^{2}
$$

We also study dynamical quantities, assuming the usual molecular dynamics (MD) involving elastic collisions between different discs and between the discs and the system boundary. The trajectories of the discs are denoted by $\boldsymbol{r}_{i}(t)$ with $t$ being the time. All of the discs have the same mass $m$ and the natural timescale $\tau$ is set by

$$
\tau=\left(\frac{m \sigma^{2}}{2 k_{B} T}\right)^{1 / 2} .
$$

Since our system exhibits rotational symmetry, the total angular momentum of the discs is conserved. In order to get rid of the trivial overall rotation of the whole system, we fix the total angular momentum of the discs as zero in all of our considerations.

\subsection{Close packing}

The upper limit of the area fraction $\eta$ is governed by close packing. Finding this closepacked area fraction $\eta_{N}^{C P}$ and the associated disc configuration for different values of $N$ constitutes an old problem of classical geometry which has not yet been solved for arbitrary $N$ [9, 10]. For small $N$ up to $N=11$, however, the close-packed configurations have been determined rigorously [11]. For $N>11$, there are some plausible guesses [12]. We have summarized all of these close-packed configurations up to $N=20$ in figure 2 . The associated close-packed area fractions $\eta_{N}^{C P}$ are shown in figure 3 .

From figures 2 and 3, one can appreciate various facts. First, as expected, there is no general simple rule for how to get the close-packed configurations for different values of $N$. They depend very sensitively on $N$, particularly for small $N$. Second, in some close-packed configurations (e.g. for $N=3,4,5,7$ ) all discs are locked, i.e. their dynamics is blocked apart from a trivial overall rotation of the total system. In others, however (e.g. for $N=8,9,13$ ), some discs are still allowed to move slightly in the close-packed configuration. A third striking fact is that there are magic numbers $N_{m}=7$ and $N_{m}=19$ associated with a close-packed configuration with curved hexagonal symmetry [10]. This implies a high packing efficiency, as demonstrated by a high value of $\eta_{N}^{C P}$. Interestingly enough, for $N_{m}-1$ (i.e. for $N=6$ and $N=18$ ), one obtains the close-packed configuration by simply removing one arbitrary disc from the magic configurations belonging to $N_{m}$. More generally, a curved hexagonal configuration fits exactly into a circle if its magic particle number has the form $N_{m}=3 i^{2}-3 i+1$ with $i=2,3,4, \ldots$. Please note, however, that for large $N_{m}$ the hexagonal configuration is not necessarily the close-packed one.

For $N \rightarrow \infty, \eta_{N}^{C P}$ approaches its bulk limit $\eta_{\infty}^{C P}=\pi / \sqrt{12} \approx 0.9069 \ldots$, which had been proven already by Gauss. The associated close-packed configuration is a hexagonal lattice. For comparison we have also shown this value in figure 3 as a dashed line. It can be seen that for $N=20, \eta_{N}^{C P}$ is still far away from the bulk limit. This is consistent with the asymptotic behaviour $\eta_{N}^{C P}=\eta_{\infty}^{C P}+\mathrm{O}(1 / N)$. 


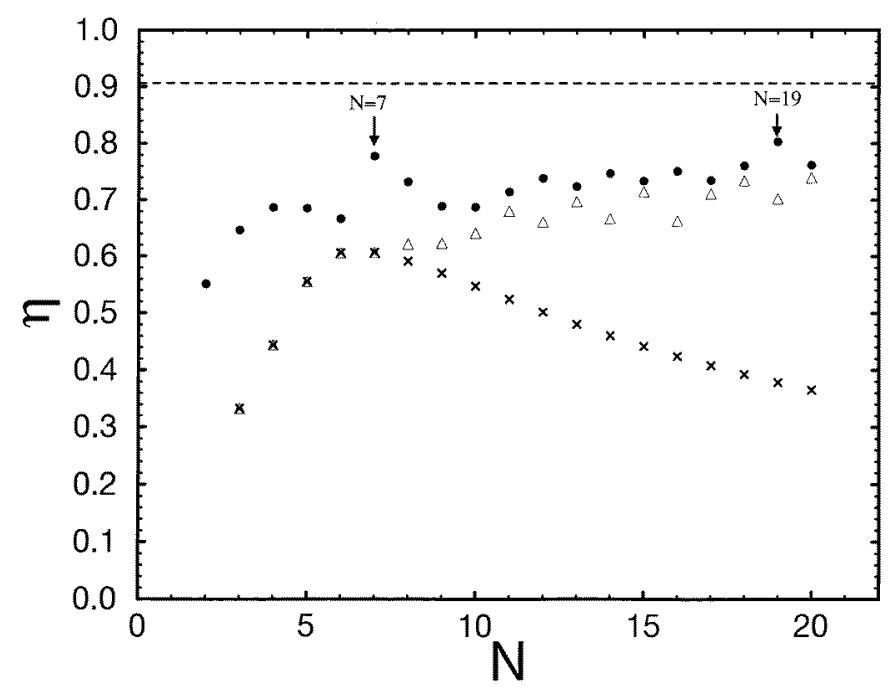

Figure 3. Different area fractions versus $N$ for $N=2-20$ : the close-packed area fraction $\eta_{N}^{C P}$ (circles); the lowest ergodic area fraction $\eta_{N}^{l}$ (crosses); the highest ergodic area fraction $\eta_{N}^{h}$ (triangles). The error for $\eta_{N}^{h}$ is smaller than the size of the symbol. The dashed line is the bulk limit for close packing, $\eta_{N}^{C P} \equiv \eta_{\infty}^{C P}=\pi / \sqrt{1} 2$.

\subsection{Ergodicity breaking}

In an ergodic system the statistical ensemble average coincides with the time average over a long system trajectory for any initial configurations with a non-zero weight in configuration space. If, for a set of given initial configurations with non-zero measure in configuration space, the system trajectory does not explore the full underlying phase space, then the system is necessarily non-ergodic. The transition from ergodic to non-ergodic behaviour is called ergodicity breaking. In the following we restrict the meaning of ergodicity somewhat, appealing to an intuitive view of the behaviour of locked close-packed configurations [13]. We call our system ergodic if, for any initial configuration with non-zero weight, any disc can reach any point of the available positional part of the phase space (involving the total accessible area $\left.\pi(R-\sigma / 2)^{2}\right)$ along a continuous trajectory. Hence ergodicity breaking implies that at least one tagged disc cannot reach all of its possible configurations within the cavity along a continuous trajectory starting from a set of given initial configurations with non-zero measure.

Keeping $N$ fixed, it is clear that for small area fractions the system is ergodic while for area fractions near close packing it becomes non-ergodic. Consequently there is at least one (but maybe several) threshold area fraction(s) marking ergodicity breaking. We call the smallest area fraction, associated with ergodicity breaking, the lowest ergodic area fraction $\eta=\eta_{N}^{l}$. In detail, $\eta=\eta_{N}^{l}$ is defined via the following property. For all area fractions which are less than or equal to $\eta_{N}^{l}$ the system is ergodic, but there is a small number $\epsilon>0$ such that the system is non-ergodic for a finite range of area fractions above $\eta_{N}^{l}$, $\eta_{N}^{l}<\eta<\eta_{N}^{l}+\epsilon$. On the other hand, the highest ergodic area fraction $\eta=\eta_{N}^{h}$ is defined as follows: the system is non-ergodic for any area fraction $\eta$ in the range $\eta_{N}^{h}<\eta \leqslant \eta_{N}^{C P}$ and there is a small number $\epsilon>0$ such that the system is ergodic for the finite range of area fractions below $\eta_{N}^{h}, \eta_{N}^{h}-\epsilon<\eta<\eta_{N}^{h}$. Clearly, $\eta_{N}^{l} \leqslant \eta_{N}^{h}$, and both ergodic area fractions $\eta=\eta_{N}^{l}$ and $\eta=\eta_{N}^{h}$ will depend on $N$, in general. 
We have applied analytical considerations [13] as well as computer simulations to determine $\eta_{N}^{l}$ and $\eta_{N}^{h}$ for different values of $N$. The results are summarized in figure 3 . For small $N$ up to $N=7$, we get $\eta_{N}^{l} \equiv \eta_{N}^{h}$ with $\eta_{N}^{l}=N / 9$ for $N=3,4,5$ and $\eta_{6}^{l}=0.6067$ and $\eta_{7}^{l}=0.6073$. One first important conclusion is that $\eta_{N}^{l}<\eta_{N}^{h}$ for $N>7$, signalling a sequence of ergodicity-breaking and ergodicity-restoring transitions for increasing area fraction.

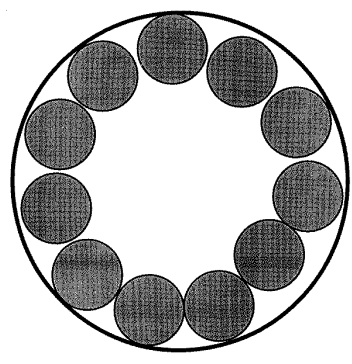

(a)

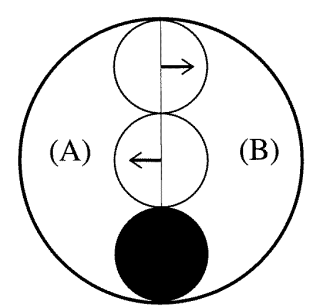

(b)

Figure 4. (a) The 'arching' effect of the particles. A ring-like disc configuration close to the wall is locked due to the convexity of the wall and due to mutual geometric confinement. (b) Determining $\eta_{N}^{h}$ for $N=3$. The tagged disc is shown as a full black circle. If $R \geqslant 1.5 \sigma$, a disc can get from the region (A) into the region (B).

We obtained the lower ergodic area fraction $\eta_{N}^{l}$ analytically for arbitrary $N$. For $N>7$, $\eta_{N}^{l}$ is governed by an arching effect shown in figure 4(a) (rigorous proof, however is still lacking). If the disc configuration is a ring close to the wall, the discs are locked due to the convexity of the wall and due to their mutual geometric confinement and cannot leave the region close to the wall. This leads to the following analytical expression for $\eta_{N}^{l}$ (valid for $N>7)$ :

$$
\eta_{N}^{l}=N\left(\frac{\sigma}{2 x+\sigma}\right)^{2}
$$

where $x$ is the smallest positive solution of

$$
(N-2) \arcsin \frac{1}{2 x}+\arcsin \frac{1}{x}-\pi=0 .
$$

Since the arching effect is a boundary phenomenon, it becomes clear that $\eta_{N}^{l}=\mathrm{O}(1 / N)$ for large $N$. In particular, $\eta_{N}^{l}$ vanishes in the bulk limit $N \rightarrow \infty$. For fixed $N$, the 'arching' effect depends crucially on the area fraction or on the cavity radius. Hence it becomes immediately clear that the system exhibits a whole scenario of transitions connected with breaking and restoring of ergodicity. This scenario becomes more and more complex for increasing $N$. Figure 4(b) shows the first four non-ergodic regimes for $N=16$ for which arching around the cavity wall happens.

The highest ergodic area fraction $\eta_{N}^{h}$ is much harder to obtain. We estimate it using a different criterion. We consider a tagged fixed disc touching the cavity boundary to eliminate rotations of the whole system; see figure 5. The straight line connecting the centre of the disc and the centre of the cavity defines two half-planes (A) and (B) corresponding to two equal areas within the cavity. There is a certain threshold area fraction above which it becomes impossible for any disc starting in area (A) to move to area (B). (The case with $N=3$ is sketched in figure 5.) For $N>2$, this area fraction is expected to be identical to $\eta_{N}^{h}$. Following this route, we determined $\eta_{N}^{h}$ by making obvious guesses for the cases with 


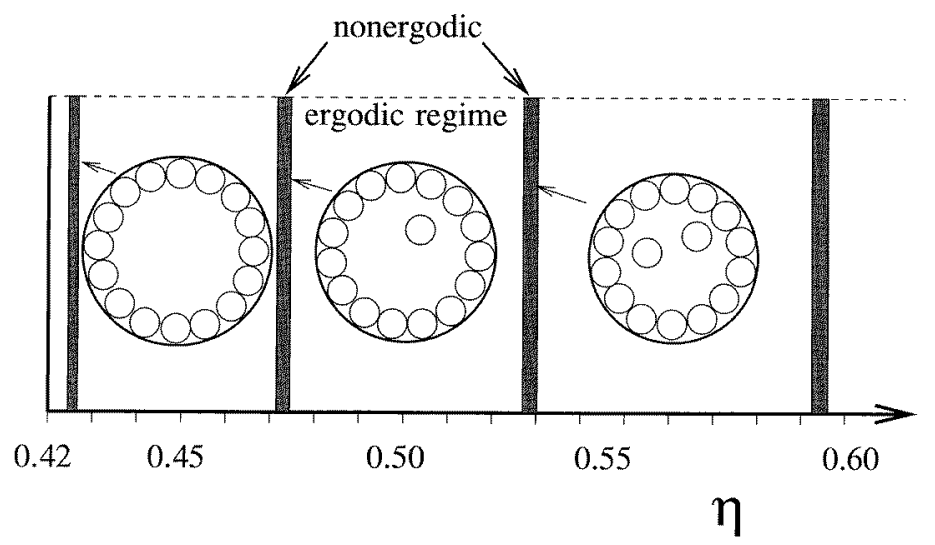

Figure 5. The first four non-ergodic regimes for which arching occurs around the wall for $N=16$.

$N=3-8,14,15,17,19$ and 20 and long MD simulations for $N=9-13,16$, and 18 . We checked our guesses by carrying out simulations as well, which allowed us to estimate the error of our pure simulation results. We finally note that $\eta_{N}^{h} \rightarrow \eta_{\infty}^{C P}$ for $N \rightarrow \infty$.

As can be seen from figure 3 , the lower ergodic area fraction $\eta_{N}^{l}$ depends smoothly and monotonically on $N$, while $\eta_{N}^{h}$ shows a subtle and strong $N$-dependence. Neither of them scales with the close-packing area fraction, i.e. they are not proportional to $\eta_{N}^{C P}$ with a universal $N$-independent prefactor. Conversely, $\eta_{N}^{h}$ is small when $\eta_{N}^{C P}$ is large, as in the case of magic numbers. If the close-packed configuration is 'open' (as e.g. for $N=10,11$ ), then the difference between $\eta_{N}^{C P}$ and $\eta_{N}^{h}$ is small.

\section{Static properties}

We now turn to statistical averages in order to work out whether there are 'fingerprints' of the bulk freezing transition in our finite confined system. Using molecular dynamics simulations, we calculated the equilibrium radial one-particle density distribution $\rho(r)$ as well as the wall pressure $P_{w}$. The radial density profile $\rho(r)$ is defined in the canonical ensemble via

$$
\rho(r)=\left\langle\frac{1}{2 \pi r} \sum_{i=1}^{N} \delta\left(r-r_{i}\right)\right\rangle
$$

where $\langle\cdots\rangle$ denotes a static canonical average of the finite system:

$$
\langle\cdots\rangle=\frac{1}{Z_{N}} \int \mathrm{d} r_{1}^{2} \cdots \int \mathrm{d} r_{N}^{2} \cdots\left[\prod_{i<j=1}^{N} \Theta\left(\left|\boldsymbol{r}_{i}-\boldsymbol{r}_{j}\right|-\sigma\right)\right]\left[\prod_{n=1}^{N} \Theta\left(R-\frac{\sigma}{2}-\left|\boldsymbol{r}_{n}\right|\right)\right] .
$$

Here the prefactor $Z_{N}$ ensures correct normalization $(\langle 1\rangle=1)$ and $\Theta(x)$ is the unit step function. The radial probability density for finding one disc at a distance $r$ from the origin is given by $2 \pi r \rho(r)$.

One possible definition of the wall pressure in a finite system is

$$
P_{w}=-\left.\frac{\partial F}{\partial A^{P}}\right|_{T, N}
$$


where $F=-k_{B} T \ln \left(Z_{N} / \Lambda^{2 N} N\right.$ !) is the (canonical) Helmholtz free energy of the finite system, $\Lambda$ denoting the (irrelevant) thermal de Broglie wavelength of the discs. Furthermore, $A^{P}=\pi(R-\sigma / 2)^{2}$ is the area which is accessible to the disc centres. It can easily be shown that, similarly to in the bulk case [14], the wall pressure is related to in the contact value of the density profile:

$$
P_{w}=k_{B} T \rho\left(R-\frac{\sigma}{2}\right) .
$$

We performed MD simulations for various magic numbers of discs, varying the area fraction. By a repeated changing of the disc velocities [15], we ensure that our averages are in the canonical rather than in the microcanonical ensemble. Our equilibration period involved typically $10^{3}-10^{4}$ collisions per disc. We started from the close-packed configurations and scaled the disc diameters in order to preserve the imposed area fraction. If the system is non-ergodic, certain regions of the configurational space are not explored by the disc trajectories. Therefore our average does not include the whole configuration space in this case. The full configurational average will exhibit 'spikes' in $\rho(r)$ at certain area fractions, signalling the threshold of ergodicity breaking and restoring. Although this effect is interesting, we shall not focus on it, due to the difficulty of accessing it numerically. The amplitude of the spikes is very small and it is difficult to do a proper sampling in a computer simulation.

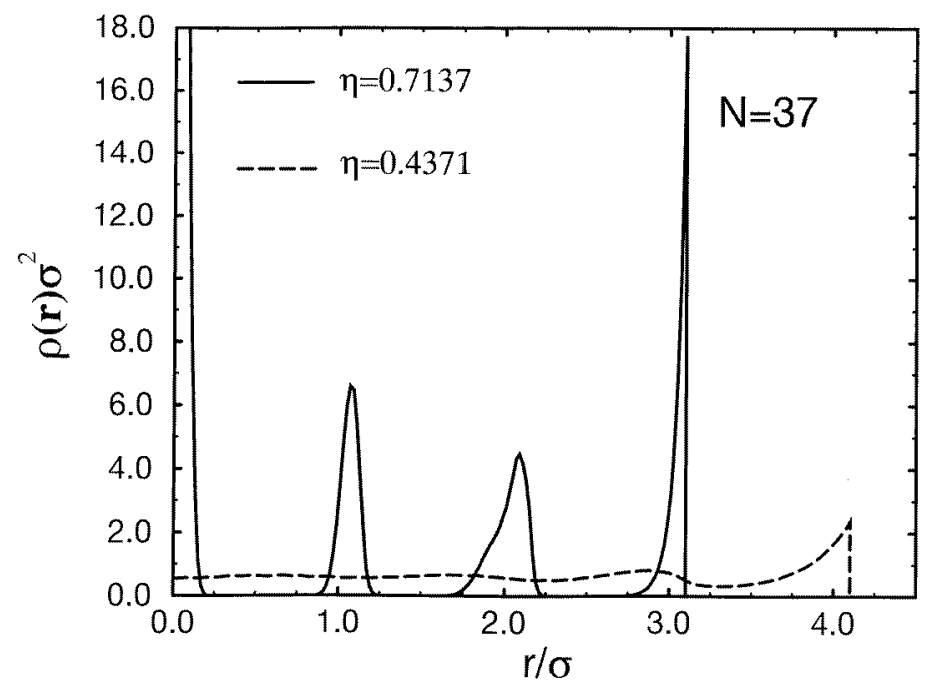

Figure 6. Density profiles $\rho(r) \sigma^{2}$ for $N_{m}=37$ discs for the area fractions $\eta=0.437$ (dashed line) and $\eta=0.7137$ (solid line). The reduced maximal distances from the origin, $R / \sigma-1 / 2$, are 3.1 and 4.1 respectively.

Our results for $\rho(r)$ are shown in figure 6. Here, we have chosen a magic number $N_{m}=37$ and two different area fractions, namely $\eta=0.437$ and $\eta=0.7137$. The former area fraction is moderate but already higher than $\eta_{37}^{l} \equiv 0.22604$, while the latter is higher but still smaller than $\eta_{37}^{h}$. As expected, the density profile is strongly inhomogeneous and exhibits different peaks corresponding to the layering of discs in concentric circular shells induced by the cavity wall. The peak heights increase practically continuously with $\eta$. One could try to define solid-like and fluid-like density profiles by exploiting the bulk Lindemann 
criterion [16]: If the relative width of the peaks is $10 \%$ of their mutual distance, then the profile is solid-like. By this classification, the first area fraction would correspond to a 'fluid-like' situation and the higher one to a 'solid-like' situation. However, as can be seen from figure 6, the widths of the peaks depend on their distances $r$ and different circular shells may coalesce into one asymmetric peak, e.g. for $r=2 \sigma$. Hence, though giving a general feeling, the Lindemann rule is not a precise and unique way to define localization in our finite system. Two further properties can be deduced from figure 6. First, the central disc gives rise to a strongly localized peak which is not fully shown. Second, the density profiles strongly pile up near the cavity wall. We finally remark that for the threedimensional counterpart of our model (hard spheres in spherical cavities), similar but only fluid-like density profiles have been discussed by other authors [17-19].

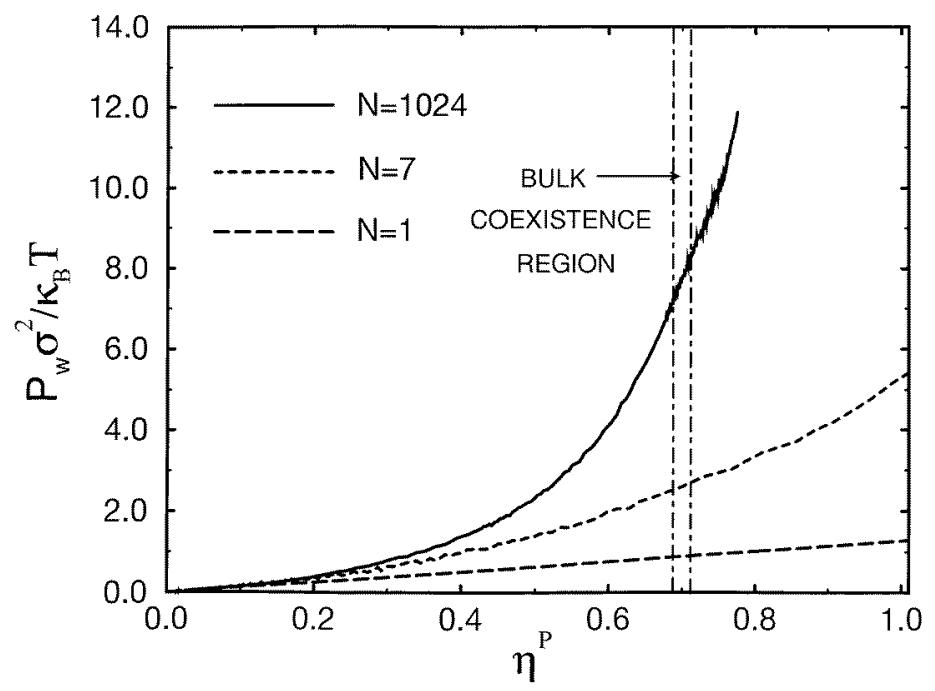

Figure 7. The equation of state, $P_{w}$ versus the physical area fraction $\eta^{P}=\sigma^{2} /\left(4(R-\sigma / 2)^{2}\right)$, for systems containing $N_{m}=7$ and $N_{m}=1027$ discs. The area fractions associated with the bulk freezing transition are also shown. The exact result for $N=1$ is the solid line. The scattering of the lines gives a rough estimate of the statistical error of the simulation results.

Results for the equation of state (wall pressure $P_{w}$ versus area fraction) are presented in figure 7 for two different magic numbers $N_{m}=7$ and $N_{m}=1027$. In our MD simulation, we calculated $P_{w}$ from the time-averaged momentum exchange of the discs with the wall during a collision. Since $\rho(r)$ strongly piles up near the wall, this procedure is much more accurate than using an expression for which one has to extrapolate the density profile. In the bulk limit $(N \rightarrow \infty)$, the equation of state $P_{w}(\eta)$ exhibits a horizontal plateau in between the two area fractions $\eta_{f}=0.69$ and $\eta_{s}=0.711$ indicating the first-order freezing transition from the fluid state to the hexagonal crystal [8]. These bulk coexisting area fractions are also shown in figure 6. Comparing the different curves, one can see that the actual results for the wall pressure in reduced units of $k_{B} T / \sigma^{2}$ depend on $N$. For comparison, we have also included the exact result for $N=1, P_{w} \sigma^{2} / k_{B} T=4 \eta^{P} / \pi$ (where $\eta^{P}=\left(N \pi \sigma^{2}\right) / 4 A^{P}$ ), in figure 7. However, even for $N=1027$ there is no horizontal plateau visible in between the bulk coexisting area fractions. This implies that we are still far away from the bulk limit. There is no clear 'fingerprint' of the bulk freezing transition in the equation of state of the finite system. 


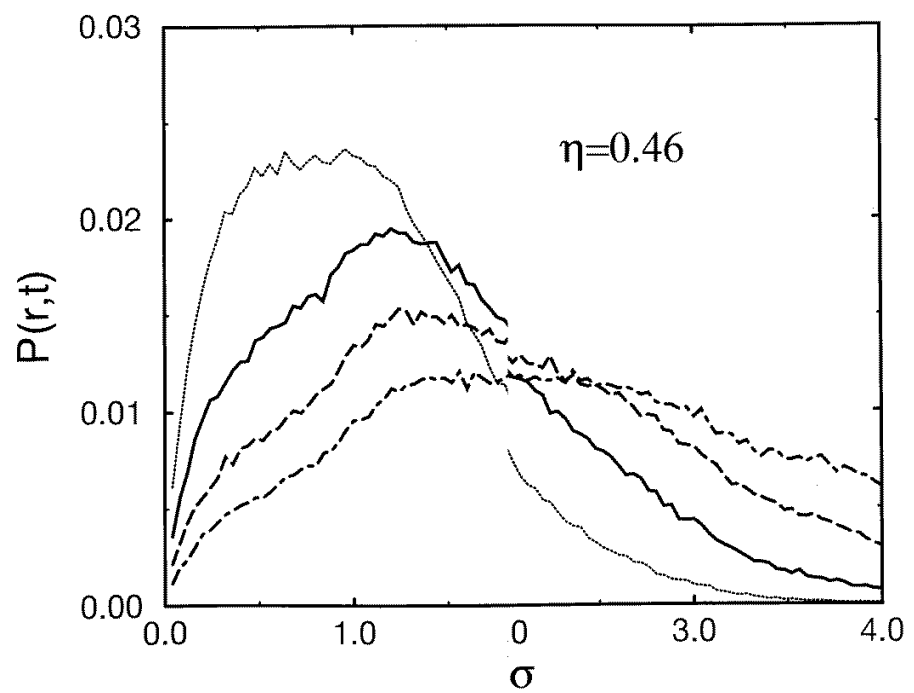

(a)

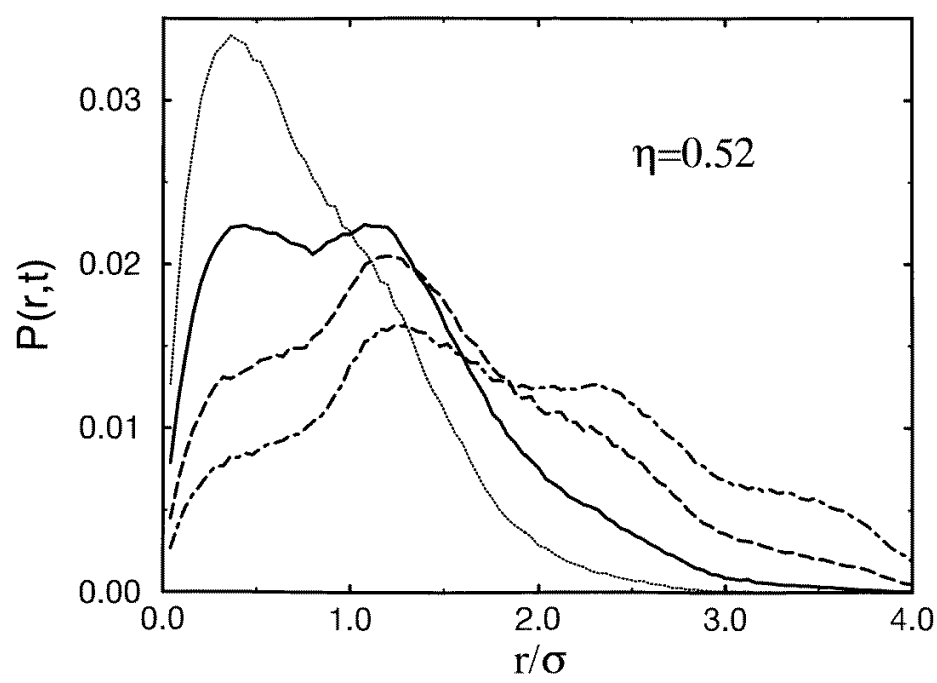

(b)

Figure 8. The probability distribution $P(r, t)$ versus the reduced distance $r / \sigma$ for $N=19$ and for four different times $t: t / \tau=15$ (dotted line), $t / \tau=30$ (solid line), $t / \tau=60$ (dashed line), $t / \tau=120$ (chain line), (a) below freezing, $\eta=0.464$, (b) at freezing, $\eta=0.52$, (c) above freezing, $\eta=0.661$. (d) The probability distribution $P(r, t)$ versus the reduced distance for $t / \tau=30$ for three different densities: $\eta=0.50, \eta=0.52$ and $\eta=0.54$. The abrupt change of the dynamical behaviour defines the density gap associated with the transition.

Summarizing, there is no obvious manifestation of the bulk freezing transition in the static quantities considered. One perhaps has to consider more sophisticated structural correlations as bond-orientational order parameters. We shall not follow this path, although it may be promising, but we show below that dynamical correlations provide much more sensitive quantities in defining the freezing transition in a finite system. 


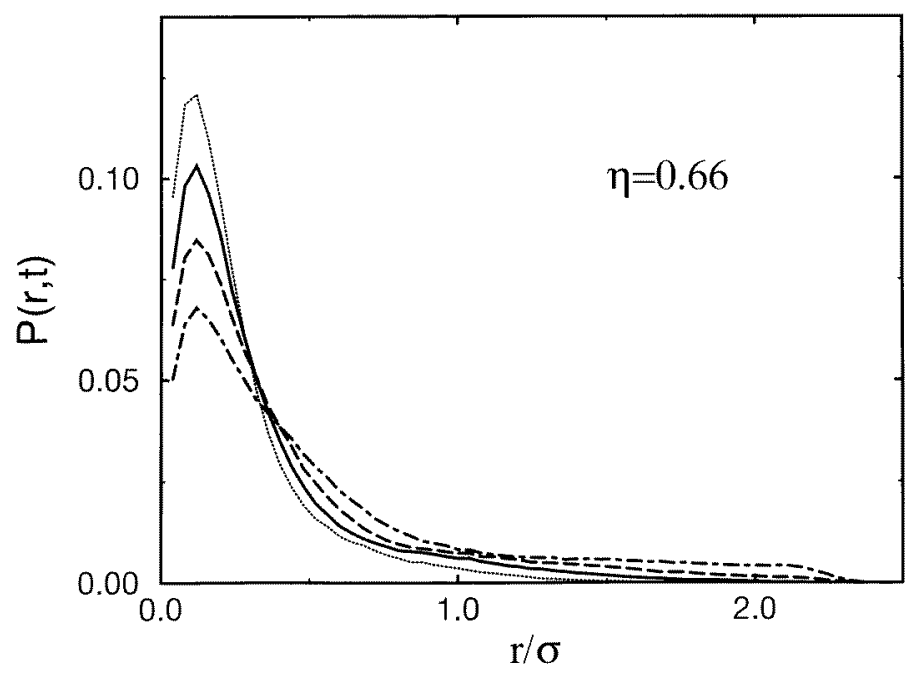

(c)

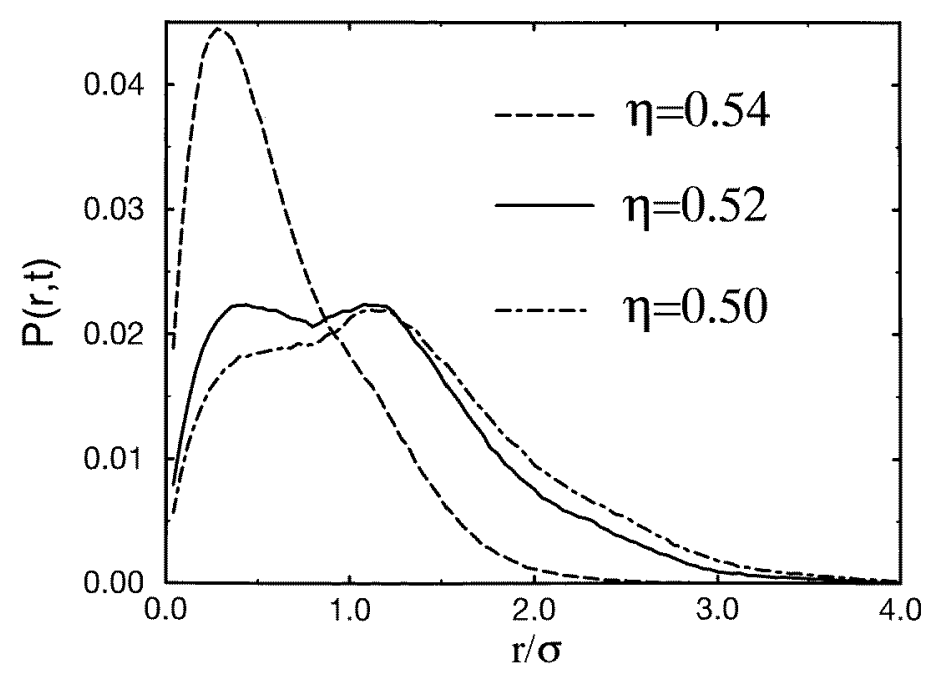

(d)

Figure 8. (Continued)

\section{Locating the freezing transition by dynamical diagnostics}

Dynamical correlations are usually measured in terms of the van Hove correlation function [20]. In particular, its self-part is the key quantity for characterizing the kinetic glass transition [21]. We consider circular averages of the van Hove function in our finite system in order to reduce the number of variables. We define the circularly averaged self-part of the van Hove function as follows:

$$
\bar{G}(r, t)=\frac{1}{N} \int \mathrm{d}^{2} r^{\prime} \delta\left(r-r^{\prime}\right)\left\langle\sum_{i=1}^{N} \delta\left(\boldsymbol{r}^{\prime}-\left[\boldsymbol{r}_{i}(t)-\boldsymbol{r}_{i}(0)\right]\right)\right\rangle .
$$


Here, $\langle\cdots\rangle$ is a canonical average over all initial conditions $\left\{\boldsymbol{r}_{i}(0)\right\}$. By definition, $\bar{G}(r, t)$ only depends on two variables, the radial distance $r$ and the time $t$. The radial probability of finding a disc after a time $t$ at a radial distance $r$ with respect to its original position at $t=0$ is given by $P(r, t)=2 \pi r \bar{G}(r, t)$.

Let us first discuss the circularly averaged van Hove functions near the bulk freezing transition. In the isotropic bulk fluid phase, the van Hove function coincides with its spherical average. After a microscopic relaxation time, $\bar{G}(r, t) \equiv \bar{G}_{f}(r, t)$ is roughly described by the solution of the two-dimensional diffusion equation

$$
\bar{G}(r, t) \sim \frac{1}{t} \exp \left(-r^{2} / 4 D t\right)
$$

where $D$ is the long-time self-diffusion coefficient which depends logarithmically on time in two dimensions [22]. In the bulk crystalline phase, on the other hand, the dynamics is very slow, and $\bar{G}(r, t) \equiv \bar{G}_{s}(r, t)$ shows a frozen peak at the origin for fixed time $t$. For very long times, vacancy diffusion sets in and leads to the build-up of a shoulder or a secondary peak in $\bar{G}(r, t)$ at $r \approx \sigma$. In the bulk coexistence region, i.e. for $\eta_{f} \leqslant \eta \leqslant \eta_{s}$, the result for the total $\bar{G}(r, t)$ is a linear superposition of $\bar{G}_{f}(r, t)$ and $\bar{G}_{s}(r, t)$ :

$$
\bar{G}(r, t)=\frac{\eta-\eta_{f}}{\eta_{s}-\eta_{f}} \bar{G}_{f}(r, t)+\frac{\eta_{s}-\eta}{\eta_{s}-\eta_{f}} \bar{G}_{s}(r, t) .
$$

Consequently, in the bulk coexistence region, the dynamics changes abruptly from hydrodynamic relaxation to some kind of vacancy diffusion manifesting itself in a shoulder at $r \approx \sigma$. Therefore we are looking for such a shoulder in our finite system.

Computer simulation results for $P(r, t)$ are presented in figures $8($ a) $-8($ d) for $N=19$ and for different area fractions $\eta$ ranging between $\eta=0.464$ and $\eta=0.66$. Clearly, the dynamics of the system becomes different upon this relatively small change in area fraction occurring. While, for $\eta=0.464$, this is similar to hydrodynamic relaxation, the location of the first peak practically stays frozen even for $\eta=0.5$. Slightly above this density, there is a clear build-up of a secondary peak at $r \approx \sigma$ (see figure 8(b), $\eta=0.52$ ) for intermediate times, which we attribute to some kind of particle exchange hopping or vacancy diffusion. For confined systems with finite $N$ this secondary peak becomes very strong such that, for longer times, its amplitude can become even higher than that of the first peak. Above $\eta=0.6$ the system behaves like a solid. The second peak grows extremely slowly with time and it vanishes for $\eta \geqslant \eta^{l}$ (figure 8(c)). As we can see in figure 8(d), the relevant change in the dynamics takes place (for $N=19$ ) in a quite narrow density gap between the hydrodynamical $(\eta \leqslant 0.5)$ and solid-like $(\eta \geqslant 0.6)$ limits. However, the width of the gap depends on $N$; it shrinks and shifts to higher values of $\eta$ as the system becomes larger and it turns into the coexistence region in the bulk limit. This behaviour suggests that a shift in the density with changing $N$ is indeed a consequence of the strong confinement. For this reason it is straightforward to define the freezing of a finite confined system within this density range by choosing an appropriate time value for $P(r, t)$ and exploiting the exotic behaviour of the second peak in this region as follows. If, after a time $t \equiv 30 \tau$, the two peaks of $P(r, t)$ have the same amplitude, then we call the associated area fraction the freezing area fraction $\eta_{N}^{f}$. Although this choice of $t(=30 \tau)$ is slightly arbitrary, the construction ensures a good location of the transition point within the density range, where important changes in the dynamics happen. A further advantage of this definition is that it is sensitive to a change in area fraction; see again figure 8(d). A situation directly at the point of the freezing transition is shown in figure 8(b).

The physical interpretation of the freezing transition implies that the associated dynamics contains a large number of particle exchange processes after a time which is roughly one 


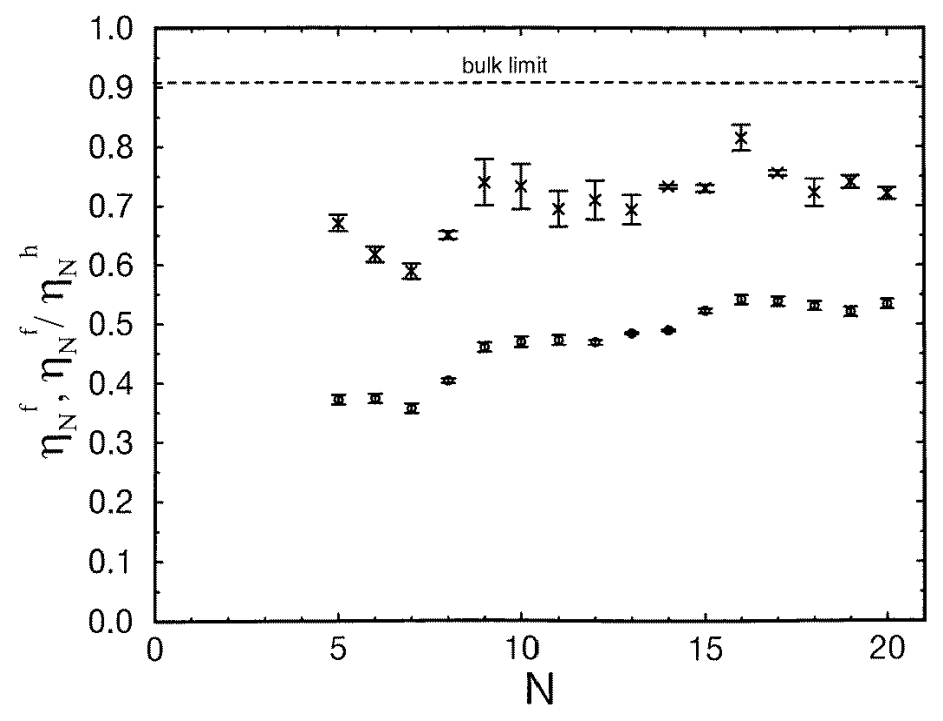

Figure 9. Freezing area fractions $\eta_{N}^{f}$ (open circles) and scaled freezing area fractions $\eta_{N}^{f} / \eta_{N}^{h}$ (crosses) versus $N$ for $N=5-20$. The statistical error is also shown.

order of magnitude larger than the microscopic timescale $\tau$.

We now discuss the $N$-dependence of $\eta_{N}^{f}$ in more detail. In figure 9 , we have plotted $\eta_{N}^{f}$ for $N=5-20$. A significant $N$-dependence can be seen. Obviously, $\eta_{N}^{f} \leqslant \eta_{N}^{h}$. It is tempting to relate our data to these ergodicity-breaking densities: one might conjecture that the change in the dynamics is related to ergodicity breaking and hence one would expect $\eta_{N}^{f}$ to scale with $\eta_{N}^{h}$, i.e. the ratio $\eta_{N}^{f} / \eta_{N}^{h}$ to be independent of $N$. We have also included this ratio in figure 9. However, it is not constant over the range of $N$ considered. The important conclusion is that the dynamics of a strongly confined system is not completely governed by ergodicity conditions. The statistical weights of all configurations also crucially determine the importance of particle exchange processes. Finally we have investigated the behaviour of $\eta_{N}^{f}$ for large $N$. In figure 10 we have plotted the results versus $1 / N$ in order to extract directly the bulk limit $N \rightarrow \infty$. Data for the magic numbers $N_{m}=1027,547,397,217,127$ and 61 are shown. They seem to converge slightly below the bulk coexistence region for $N \rightarrow \infty$. Another important conclusion is that freezing occurs for a smaller area fraction than for the bulk. Hence, the confinement slows the dynamics down. Moreover, this proves a posteriori that our dynamical definition of the freezing densities made sense and that the particle exchange processes are indeed the dynamical 'fingerprint' of the bulk freezing transition. It also demonstrates that there is no kinetic glass transition for hard discs, which would be the natural bulk limit for frozen dynamical correlations. This is consistent with earlier findings [24].

\section{Conclusions}

To summarize: we studied a toy model of hard discs in a circular cavity and found that the bulk freezing transition manifests itself as a dynamical crossover from hydrodynamic relaxation to particle exchange hopping. The corresponding crossover area fractions are not directly related to ergodicity breaking for the system. 


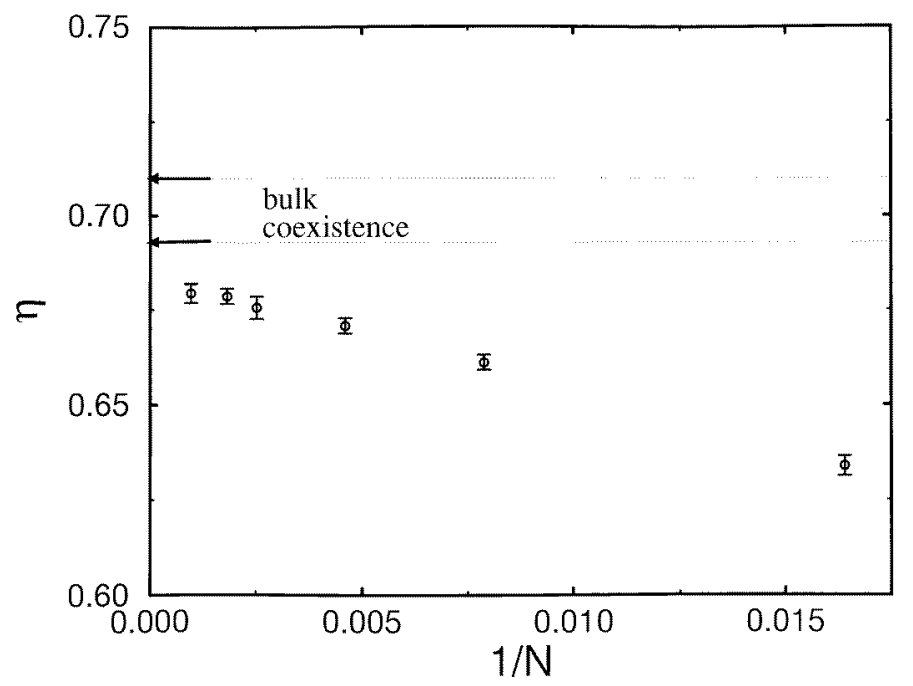

Figure 10. Freezing area fractions $\eta_{N}^{f}$ (open circles) together with their statistical error versus $1 / N$ for $N=1027,547,397,217,127$ and 61 . The bulk coexistence region is also shown.

We emphasize that freezing in a finite system can mimic both a bulk crystallization and a bulk glass transition [23]. In the simple two-dimensional model that we studied, a randomclose-packed configuration is unstable and immediately collapses to a hexagonal crystal [24]. This might change in three spatial dimensions, for which a bulk glass transition is possible [25]. The glass transition is frequently understood as being accompanied by ergodicity breaking [26]. Therefore it would be interesting to study our model in three dimensions.

Let us now relate our results to experiments. The best realizations of our model are sterically stabilized colloids confined to a two-dimensional circular cavity [27]. In principle it is possible to prepare such samples. The additional advantage is that one can investigate the dynamics of the system in real space by video-microscopy. Up to now, only magnetic colloids have been confined in two-dimensional cavities of defined shape [7]. They interact, however, via a softer potential $\propto 1 / r^{3}$. Describing them in terms of hard discs is an extreme approximation. Thus it would be interesting to realize the model for harsher colloidal interactions and to check our theoretical predictions for the ergodicity properties and the dynamics.

Another experimental realization concerns fluids in porous media. In this case, the cavities are three dimensional. In a typical experiment one averages over different pore sizes and shapes. Hence a quantitative comparison becomes more difficult. The qualitative features, however, should be similar. Our result that the freezing has already occurred at smaller densities, as compared to the case for the bulk, seems to contradict recent experimental findings for the glass transitions in porous media [5, 6], which were found to occur at lower temperatures than in the bulk. However, there are several caveats. First, we are talking about densities, while in the experiments the density inside the pores is not known and only the temperature can be controlled. Second, in our theory, we have excluded the trivial whole-system rotational modes, which can contribute significantly to speeding up the total molecular dynamics. Third, the particles considered in the experiment are complex organic molecules which may behave differently to hard discs. Last, the roughness, shape and connectivity of the cavity wall are more complicated in porous media than in our 
model. We conclude that in our system the confinement slows the dynamics down rather than accelerating it.

We finish with three remarks. First, we hope that our results for the static properties which are the key quantities in classical density functional theories [16] will stimulate more research in this direction. In particular, it would be interesting to apply Rosenfeld's density functional [28], which incorporates packing effects exactly, to situations of solid-like density profiles, as was done for three dimensions for the liquid-like region [18]. Second, although, our mathematical assumptions concerning ergodicity are quite plausible conjectures, they are not at the level of a rigorous proof. We hope that the demands of the physical applications of these unsolved problems will motivate mathematical research in this field of classical geometry. Third, we would like to draw attention to the difference between our boundary conditions and the periodic boundary conditions frequently used in computer simulations to avoid finite-size effects. In fact, even for large particle numbers $N>1000$, our results have shown that the system is still far away from the bulk limit. This is due to the massive influence of the hard cavity wall. For periodic boundary conditions, the system is much closer to the bulk limit for such a particle number.

\section{Acknowledgments}

We thank F Kremer, J Jäckle, S Neser, S-C Kim, A Heuer and M Schmidt for helpful remarks.

\section{References}

[1] Rowlinson J S 1986 J. Chem. Soc. Fraday Trans. II 821801

Radhakrishnan R and Gubbins K E 1997 Phys. Rev. Lett. 792847

Roman F L, White J A and Velasco S 1996 Phys. Rev. E 532360

Suh S-H, Rho S-B and Kim S-C 1993 J. Chem. Eng. Japan 26431

Hüller A 1994 Z. Phys. B 93401

Hüller A 1994 Z. Phys. B 9563

[2] Klafter J and Drake J M (ed) 1989 Molecular Dynamics in Restricted Geometries (New York: Wiley)

[3] Schmidt M and Löwen H 1997 Phys. Rev. E 557228

[4] Fehr T and Löwen H 1995 Phys. Rev. E 524016

[5] Arndt M, Stannarius R, Groothues H, Hempel E and Kremer F 1997 Phys. Rev. Lett. 792077

Arndt M, Stannarius R, Gorbatschow W and Kremer F 1996 Phys. Rev. E 545377

Gorbatschow W, Arndt M, Stannarius R and Kremer F 1996 Europhys. Lett. 35719

[6] Pissis P, Daoukaki-Diamanti D, Apekis L and Christodoulides C 1994 J. Phys.: Condens. Matter 6 L325

[7] Bubeck R, Neser S, Bechinger C and Leiderer P 1998 Prog. Colloid Polym. Sci. at press

[8] Weber H, Marx D and Binder K 1994 Europhys. Lett. 27593

For a recent compilation and discussion of different data, see

Mitus A C, Weber H and Marx D 1997 Phys. Rev. E 556855

[9] See, e.g.,

Fejes-Tóth L 1972 Lagerungen in der Ebene auf der Kugel und im Raum (Berlin: Springer)

[10] Lubachevsky B D and Graham R L 1997 Discrete Comput. Geom. 18179

[11] Melissen H 1994 Geom. Dedicata 5015

[12] Kravitz S 1967 Math. Mag. 4065

Goldberg M 1971 Math. Mag. 44134

Pirl U 1969 Math. Nachr. 40111

[13] We remark that none of our ergodicity considerations are rigorous mathematical statements, but they are based on plausible analytical arguments and computer simulations.

[14] Fisher I Z 1964 Statistical Theory of Liquids (Chicago, IL: Chicago University Press)

[15] Heyes D M 1993 Chem. Phys. 82285

[16] Löwen H 1994 Phys. Rep. 237249 
[17] Macpherson A K, Carignan Y P and Vladimiroff T 1987 J. Chem. Phys. 871768

[18] Gonzalez A, White J A, Román F L, Velasco S and Evans R 1997 Phys. Rev. Lett. 792466

[19] Chui S T 1990 Phys. Rev. B 4311523

[20] Hansen J-P and McDonald I R 1986 Theory of Simple Liquids 2nd edn (London: Academic)

[21] Barrat J L and Klein M 1991 Annu. Rev. Phys. Chem. 4223

[22] Alder B and Wainwright T 1970 Phys. Rev. A 118

[23] Nayak S K, Jena P, Ball K D and Berry R S 1998 J. Chem. Phys. 108234

[24] Berryman J G 1983 Phys. Rev. A 271053

[25] Doliwa B and Heuer A 1998 Phys. Rev. Lett. 804915

[26] Jäckle J 1996 Phys. Bl. 52351

Donati C and Jäckle J 1996 J. Phys.: Condens. Matter 82733

Jäckle J 1998 Prog. Theor. Phys. Suppl. at press

[27] Dinsmore A D, Wong D T, Nelson P and Yodh A G 1998 Phys. Rev. Lett. 80409

[28] Rosenfeld Y, Schmidt M, Löwen H and Tarazona P 1996 J. Phys.: Condens. Matter 81577 Rosenfeld Y, Schmidt M, Löwen H and Tarazona P 1997 Phys. Rev. E 554245 